\title{
Different Types of Maculopathy in Eyes after a High-Voltage Electrical Shock Injury
}

\author{
Hye Rim Choe Un Chul Park
}

Department of Ophthalmology, Seoul National University Hospital, Seoul, South Korea

\section{Keywords}

Maculopathy · Electric shock

\begin{abstract}
Background: We report a case of different types of maculopathy in eyes after a high-voltage electrical shock injury. Case Report: A 43-year-old male suffered high-voltage electrical injury through his left arm. He underwent cataract surgery in both eyes 3 months after the injury, but there was no vision improvement. Ocular examination, including spectral domain optical coherence tomography, revealed diffuse retinal atrophy in the left eye which did not change until the final visit. In the right eye, an impending macular hole was observed but regressed spontaneously 9 months after the injury, and the visual acuity improved to 20/32 at the final visit. Conclusion: Two different types of maculopathy can occur in each eye after high-voltage electrical shock injury, and this might be due to asymmetric pathogenesis of the eyes according to the proximity to the route of electrical current.

(C) 2019 The Author(s) Published by S. Karger AG, Basel
\end{abstract}

\section{Introduction}

Ocular complications caused by high-voltage electrical injury are rare. The severity of injury is determined by tissue resistance and proximity to the inlet. The authors present a case of asymmetric macular complications in eyes after a high-voltage electric shock injury. 


\section{Case Report}

A 43-year-old male electrical engineer suffered a high-voltage electric shock injury $(22,000 \mathrm{~V})$. He stated that the current entered his body through his left arm, which was amputated due to severe burning. During hospitalization at an outside hospital, an ophthalmic exam was performed for the decreased vision in both eyes only after treatment at the intensive care unit, and bilateral cataract was found. He underwent cataract extraction and intraocular lens implantation in both eyes 3 months after the injury. Although cataract surgeries were uneventful, visual improvement was slight, and he was referred to our clinic for further evaluation. Because of the limited information at the referral, we could not confirm visual acuity at the time of the injury.

At the first visit, 9 months after the injury, there was a burn scar on his left face, and his left arm had been amputated. The best-corrected visual acuities (BCVA) were 20/40 in the right eye and hand motion in the left eye. There was no abnormal finding in the anterior segments, but the specular microscopy showed decreased endothelial cell count $(1,838$ cells $/ \mathrm{mm}^{2}$ ) and hexagonality (49\%) in the left eye compared to the right eye which showed a cell density of 2,463 cells $/ \mathrm{mm}^{2}$ and hexagonality of $61 \%$. Funduscopic examination showed no significant finding in the right eye (Fig. 1a), but a pigmented fibrotic lesion inferior to the pale optic disc, generalized vessel attenuation, and retinal pigment epithelial (RPE) atrophy at mid-periphery were observed in the left eye (Fig. 1b). Spectral domain optical coherence tomography (OCT) examination revealed disruption and a focal defect in the ellipsoid zone in the right macula (Fig. 2e), and there was diffuse retinal atrophy in the left eye (Fig. 2f). On fundus autofluorescence, the right eye showed a hyperautofluorescent focus at the fovea (Fig. 1c), and the left eye showed diffuse hypoautofluorescence at the maculopapillary area (Fig. 1d). On fluorescein angiogram, the left eye showed diffuse stippled hyperfluorescence corresponding to the RPE atrophy (Fig. 1f). Both eyes showed peripheral leakage in the late phase implicating vasculopathy, which was more prominent in the left eye (Fig. 1e, f).

When reviewing the previous OCT images performed at the outside hospital, the left eye image 3 months after the injury showed atrophic change comparable with the OCT image at our clinic (Fig. 2b), but the right eye showed an impending macular hole with a very thin inner retinal layer (Fig. 2a). On OCT image 5 months after the injury, the outer retinal defect in the right eye had decreased (Fig. 2c).

He was regularly followed up until 18 months after the injury. There was further improvement in ellipsoid zone integrity on the OCT image of the right eye (Fig. 2g), and BCVA had improved to 20/32, while there was no change in the left eye (Fig. $2 \mathrm{~h}$ ).

\section{Discussion}

The severity of tissue damage from electric shock is determined by tissue resistance and the proximity of the route travelled by the electrical current [1]. Posterior segment complications after electric shock injury include macular cyst, macular hole, vascular occlusions, or RPE changes $[2,3]$. The electrical current itself, thermal damage generated by the tissue resistance, and ischemia resulting from coagulation and necrosis of vasculature are the proposed pathogeneses for retinal complications after electric shock injury. The macula is more sensitive to thermal damage because the macular RPE has a high concentration of melanin granules which increase the resistance, resulting in greater thermal denaturation, and they are thicker and more tightly packed than in other areas, accumulating more thermal energy [4]. In addition, 
thermal shrinkage of the vitreous can induce posterior vitreous detachment exerting macular traction $[2,5]$.

There have been several reports of macular hole after electric shock using fundus photography, but they were mostly negative for Watzke-Allen sign [6] and likely to be impending macular holes or macular cysts instead of full-thickness macular holes [4, 7]. In a case of bilateral impending macular holes after electrical injury as firstly confirmed by OCT, one eye showed spontaneous regression with vision improvement, while the other eye showed partial regression of the outer retinal defects when followed up until 7 months after the injury [7]. However, another case of bilateral impending macular holes confirmed by OCT progressed to full-thickness holes with retinal detachment 2 months after the injury [5].

Our case shows that macular damage after electric shock can be asymmetric in the eyes, probably due to a difference in the distance from the electricity inlet. The left eye, which was closer to the inlet of the electric current, showed a more severe type of maculopathy as manifested by diffuse retinal atrophy, but the right eye showed a relatively mild form of macular damage. The main cause of irreversible atrophic change throughout the entire retina in the left eye might be direct electrical damage on neurons and vessels by the higher-intensity current that passed through the left eye. On the other hand, the current through the right eye was not so intense as to cause neural or vascular damage, but thermal denaturation over the heated RPE was the main pathogenesis for the outer macular damage which was reversible after several months with functional recovery.

\section{Conclusion}

Considering our case and the previous reports, it is recommended to observe impending macular hole or macular cyst after electrical injury with a frequent follow-up because many cases show spontaneous regression with functional improvement. However, when impending macular hole progresses to a full-thickness macular hole with or without retinal detachment, surgery with pneumatic tamponade is recommended [1].

\section{Statement of Ethics}

The patient provided oral consent for publication of this report.

\section{Disclosure Statement}

The authors have no competing interests to declare, and no financial support was received for this submission.

\section{References}

1 Ouyang P, Karapetyan A, Cui J, Duan X. Bilateral impending macular holes after a high-voltage electrical shock injury and its surgical outcome: a case report. J Med Case Reports. 2014 Dec;8(1):399.

2 Rajagopal J, Shetty SB, Kamath AG, Kamath GG. Macular hole following electrical shock injury. Can J Ophthalmol. 2010 Apr;45(2):187-8.

3 von Bahr G. Electrical injuries. Ophthalmologica. 1969;1-3(1):109-17. 


\section{Case Reports in Ophthalmology}

\begin{tabular}{l|l}
\hline Case Rep Ophthalmol 2019;10:19-23 \\
\hline DOI: 10.1159/000496196 & $\begin{array}{l}\text { @ } 2019 \text { The Author(s). Published by S. Karger AG, Basel } \\
\text { www.karger.com/cop }\end{array}$ \\
\hline
\end{tabular}

Choe and Park: Different Types of Maculopathy in Eyes after a High-Voltage Electrical Shock Injury

4 Rao KA, Rao LG, Kamath AN, Jain V. Bilateral macular hole secondary to remote lightning strike. Indian J Ophthalmol. 2009 Nov-Dec;57(6):470-2.

5 Faustino LD, Oliveira RA, Oliveira AF, Rodrigues EB, Moraes NS, Ferreira LM. Bilateral maculopathy following electrical burn: case report. Sao Paulo Med J. 2014 Dec;132(6):372-6.

6 Handa JT, Jaffe GJ. Lightning maculopathy. A case report. Retina. 1994;14(2):169-72.

7 Sony P, Venkatesh P, Tewari HK, Garg SP. Bilateral macular cysts following electric burn. Clin Exp Ophthalmol. 2005 Feb;33(1):78-80.
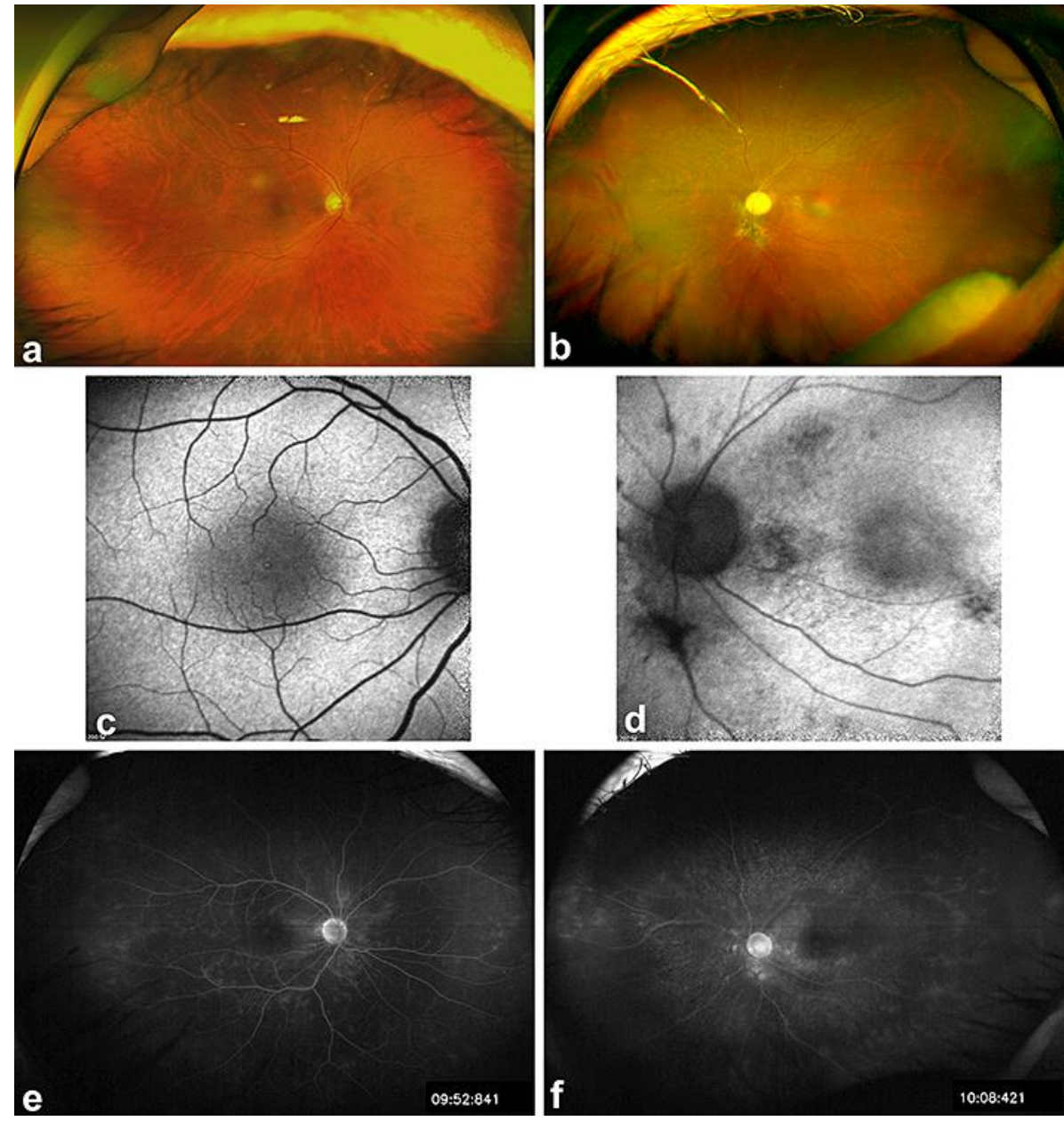

Fig. 1. a, b Ultra-wide field retinal images show no significant finding in the right eye but show a pigmented fibrotic lesion inferior to the pale optic disc, generalized vessel attenuation, and retinal pigment epithelial (RPE) atrophy at mid-periphery in the left eye. c, $\mathbf{d}$ On fundus autofluorescence images, the right eye shows a hyperautofluorescent focus at the fovea, and the left eye shows diffuse hypoautofluorescence at the maculopapillary area. e, $\mathbf{f}$ On fluorescein angiogram, both eyes show peripheral vascular leakage in the late phase, and the left eye shows diffuse stippled hyperfluorescence corresponding to the RPE atrophy. 


\section{Case Reports in Ophthalmology}

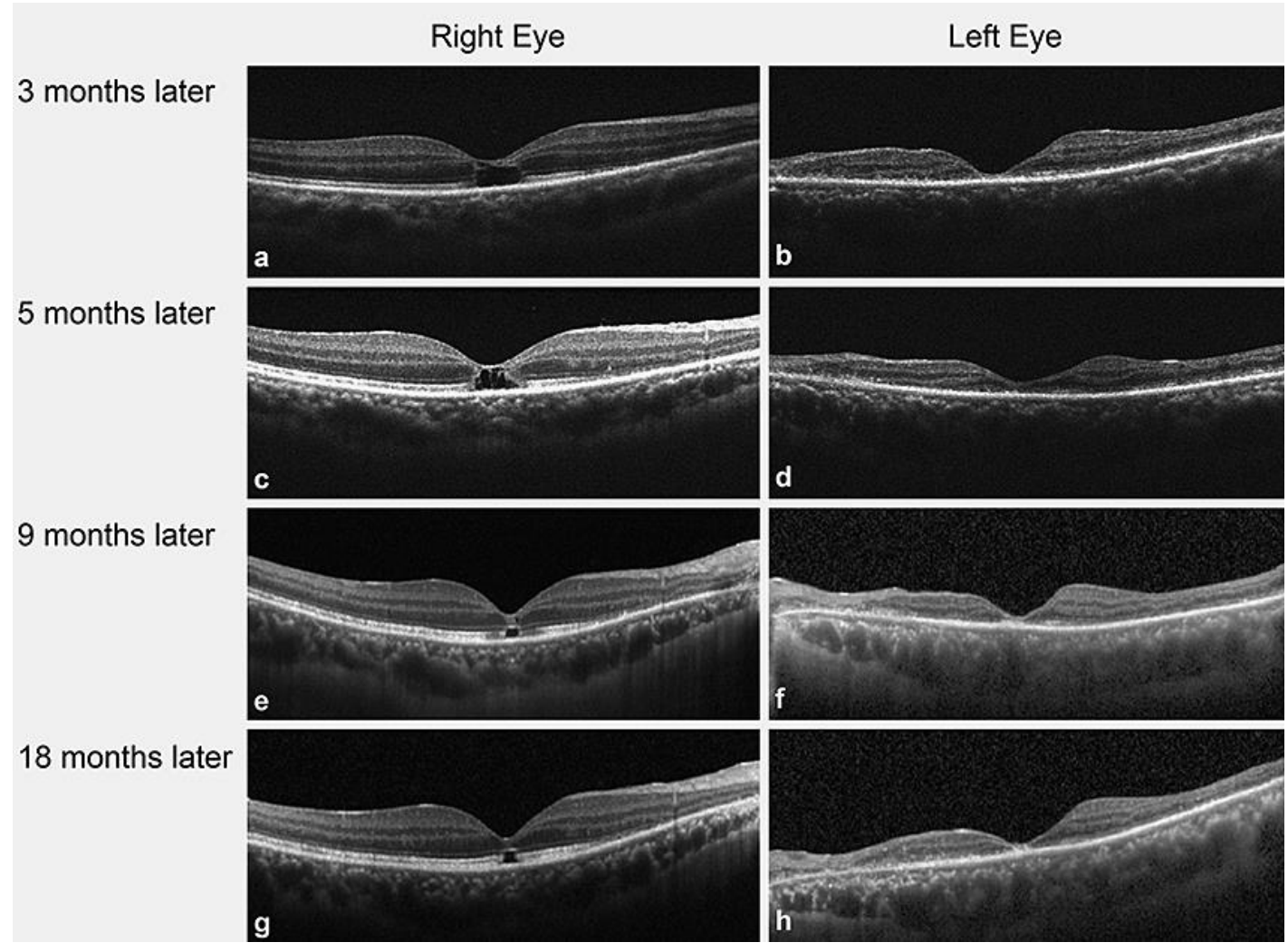

Fig. 2. Serial horizontal scans of spectral domain optical coherence tomography in both eyes. At 3 months after the injury $(\mathbf{a}, \mathbf{b})$, an impending macular hole with a very thin inner retinal layer was observed in the right eye and diffuse atrophic change with foveal thinning in the left eye. At 5 months after the injury (c, d), the outer retinal defect had decreased in the right eye. On the first visit at our clinic, 9 months after the injury (e, f), the impending macular hole was found to have regressed with only a focal defect in the ellipsoid zone with a visual acuity of 20/40. At the last follow-up, 18 months after the injury $(\mathbf{g}, \mathbf{h})$, there was improvement in ellipsoid zone integrity in the right eye, and visual acuity had improved to 20/33. However, diffuse retinal atrophy in the left eye had not recovered, and the final visual acuity was hand motion. 